\title{
Exercise training in multifocal motor neuropathy - a case report study
}

\author{
Lars K Markvardsen ${ }^{1 *}$, Kristian Overgaard ${ }^{2}$, John Vissing ${ }^{3}$ and Henning Andersen ${ }^{1}$ \\ ${ }^{1}$ Department of Neurology, Aarhus University Hospital, Aarhus \\ ${ }^{2}$ Section for Sport Science, Institute of Public Health, Aarhus University, Aarhus \\ ${ }^{3}$ Copenhagen Neuromuscular Centre, Department of Neurology, Rigshospitalet, Copenhagen
}

\begin{abstract}
Introduction: Regular physical exercise leads to improved muscle strength and aerobic capacity in chronic inflammatory demyelinating polyneuropathy, but the effect in multifocal motor neuropathy (MMN) is unknown.

Methods: Of the 15 invited MMN patients, four patients performed 12 weeks of resistance exercise with unilateral training of wrist and elbow and 12 weeks of aerobic exercise on an ergometer bike. Exercise periods were preceded by a run-in period without scheduled exercise. Primary outcomes were changes in combined isokinetic strength (cIKS) and aerobic capacity $\left(\mathrm{VO}_{2}-\mathrm{max}\right)$.

Results: Resistance exercise was well tolerated. cIKS improved at wrist by $86 \%, 18 \%$ and $16 \%$ in three patients and at elbow by and $30 \%$ and $16 \%$ in two participants, while one patient was unchanged. $\mathrm{VO}_{2}$-max was increased in two participants by 13 and $15 \%$, while two participants deteriorated by $9 \%$.

Discussion: Resistance and aerobic exercise was feasible in MMN patients. The effects varied individually from slight decrease to considerable improvements, which supports further investigations of exercise in MMN.
\end{abstract}

\begin{abstract}
Abbreviations: CIDP: chronic inflammatory demyelinating polyneuropathy; CIKS: combined isokinetic muscle strength; MMN: multifocal motor neuropathy; SCIG: subcutaneous immunoglobulin; $\mathrm{VO}_{2}$-max: maximal oxygen consumption velocity.
\end{abstract}

\section{Introduction}

Multifocal motor neuropathy (MMN) is a chronic inflammatory multifocal neuropathy presenting with asymmetrical distal muscle weakness without sensory disturbances [1]. Evidence based treatment of $\mathrm{MMN}$ is limited to immunoglobulin given intravenously or subcutaneously $[2,3]$.

In patients with inflammatory polyneuropathy, physical exercise training has been shown to improve muscle strength and aerobic capacity [4-6]. These studies included primarily patients recovering from Guillain-Barré syndrome and therefore there is a lack of information about exercise in chronic forms of inflammatory neuropathy. In a recent study, we demonstrated that muscle strength and aerobic capacity increased following 12 weeks of resistance and aerobic exercise in patients with chronic inflammatory demyelinating polyneuropathy (CIDP) [7].

So far, the effect of physical exercise has not been evaluated in patients with MMN.

\section{Methods}

\section{Study population}

Patients diagnosed with MMN, receiving maintenance treatment with subcutaneous immunoglobulin (SCIG), were eligible for inclusion in the study. Inclusion criteria were stable clinical condition and treatment with SCIG in the preceding three months, preserved gait function and age $>18$ years. Exclusion criteria were known liver or kidney disease, other immunomodulatory treatment, regular physical exercise $>1$ hour per week or $>4 \mathrm{~km}$ bicycling per day.

Participants were recruited from the outpatient clinic at the neurological departments at Aarhus University Hospital and Rigshospitalet, Copenhagen.

The study was registered at ClinicalTrials.gov (NCT02121678) and was approved by the local Ethics Committee in the Central Region of Denmark.

\section{Study design}

At enrollment, all participants were allocated to start with either aerobic or resistance exercise for 12 weeks, each followed by the other training regimen. All participants had a run-in period of 12 weeks without any scheduled exercise.

For aerobic training, participants exercised on an ergometer bike at home for 20-30 minutes, three times weekly. Based on maximal and resting heart rate from the initial $\mathrm{VO}_{2}$-max test at enrollment, the target heart rate of $70 \%$ of heart rate reserve was calculated. During each exercise session, the participants wore a pulse belt (Polar FT7, Polar

${ }^{\star}$ Correspondence to: Lars Kjøbsted Markvardsen, Department of Neurology, Aarhus University Hospital, Palle Juul-Jensens Boulevard 165, DK-8200 Aarhus N, Denmark, Tel: 00457845 4215; E-mail: larsmark@rm.dk

Key words: $M M N$, resistance training, aerobic training, muscle strength Received: July 02, 2019; Accepted: July 08, 2019; Published: July 11, 2019 
Electro Oy, Finland) that recorded the average heart rate. Adherence to training was monitored at telephone interviews every second week, combined with recordings from the heart rate monitors and diary recordings.

Aerobic capacity was determined by measuring the maximal oxygen consumption rate $\left(\mathrm{VO}_{2}\right.$-max) as described elsewhere and compared to a normal material $[8,9]$.

During the resistance training period, the participants exercised unilaterally with dumbbells with the untrained side serving as control. In all participants, resistance exercises involved wrist flexors/extensors and elbow flexors/extensors of the most involved side. The load for resistance exercise was based on determination of 12-repetition-max (12-RM). Supervised by one of the authors (LKM), each participant used dumbbell to determine 12-RM. The training program used has been described elsewhere [7]. The weakest side was chosen for training. In case both sides were affected, the non-dominant side was chosed.

Combined isokinetic muscle strength (cIKS) was evaluated by dynamometry (BIODEX System 3, Biodex Medical Systems Inc., Shirley, NY, USA) as described elsewhere. Shortly, muscle strength of elbow and wrist flexion/extension are measured while the patient uses his/her maximal performance. The peak torque of eight repetitions were used. The sum score of wrist flexion/extension and elbow flexion/ extension were calculated and compared to a normal material [10].

\section{Statistics}

For the aerobic and resistance training, the primary outcomes were changes in maximal oxygen consumption $\left(\mathrm{VO}_{2}\right.$-max) and cIKS at wrist and elbow, respectively.

Due to the low number of participants statistical analyses were not performed. Consequently, all data presented in the text are medians (min to max).

\section{Results}

Fifteen patients with MMN were invited to participate. Two of them fulfilled an exclusion criterion, nine declined to participate, leaving four patients who could be included. The main reason for this was that many patients found participation in the study time consuming. The median duration of MMN was 16 years prior to inclusion ( 6 to 32 ), and they had been treated with SCIG for 6.8 years ( 3 to 10 ) with a mean dose of $25.6 \mathrm{~g} /$ week (18 to 30 ) and unchanged for more than 12 months for all participants.
From heart rate monitors and diaries, we found that the participants on average exercised 2.5 times per week. The median heart rate during aerobic exercise was 140 beats per minute (132 to 150), which was 101 $\%$ (98 to 108) of the target heart rate. The 1-repetition max increased by $70 \%$ (32 to 102$)$.

At enrollment all patients had a lower muscle strength of wrist and elbow (flexion and extension) and aerobic capacity when compared to a normal material with respect to gender, age and body weight (Table 1).

During the run-in period, cIKS was overall changed by $2.8 \%(-4.8$ to 6.7 ) on the side chosen for future training and $-1.5 \%(-8.9$ to 4.0$)$ on the control side. In the same period, aerobic capacity changed by $3.4 \%$ (-31 to 5.3).

At the wrist, cIKS increased in three patients by $86.0 \%$ (\#1), 19\% (\#3) and $16 \%(\# 4)$, whereas one patient decreased by $8 \%(\# 2)$ (Figure $1 \mathrm{~A})$. At the elbow, cIKS also increased in three patients by $30 \%(\# 1)$, $16 \%(\# 3)$ and $7 \%(\# 4)$ and decreased in one patient by $8 \%(\# 2)$ (Figure 1B). On the control side, muscle strength was increased by $13.6 \%$ (\#1) and $18.3 \%$ (\#3) or unchanged $(-6.0 \%(\# 2)$ and $-5.3 \%(\# 4))$. This could be due to a low muscle strength before initiation of exercise in this patient compared to the other three (Table 1). In contrast, the other three patients did not experience any significant changes.

During aerobic training, we found that aerobic capacity on average was unaltered. Thus, two patients experienced an increase in aerobic capacity of $13 \%(\# 4)$ and $15 \%$ (\#3), whereas the two other patients had a decrease of $9 \%$ (\#1 and \#2) (Figure 1C).

One patient (\#2) complained of lower back pain during aerobic training and withdrew consent after 8 weeks of training although the patient completed the resistance training. None of the participants had any side effects to resistance training.

\section{Discussion}

In this small study of resistance and aerobic training in MMN, we did not find a consistent improvement of muscle strength or aerobic capacity after training.

The effect of training has not been evaluated earlier in MMN. In a recent study from our group, using the same training protocols, we demonstrated a positive effect of resistance and aerobic training in patients with CIDP. Among these patients, an increase of muscle strength of $14 \%$ following unilateral resistance training and an increase of aerobic capacity of $11 \%$ following aerobic training was statistically significant [7].

Table 1. Baseline characteristics and changes in isokinetic muscle strength (cIKS) and aerobic capacity $\left(\mathrm{VO}_{2}\right.$-max) for each of the four MMN patients included

\begin{tabular}{|c|c|c|c|c|}
\hline Patient & $\# 1$ & $\# 2$ & $\# \mathbf{3}$ & $\# 4$ \\
\hline Sex & $\mathrm{F}$ & M & $\mathrm{F}$ & $\mathrm{F}$ \\
\hline Age & 60 & 45 & 67 & 63 \\
\hline Duration of MMN (years) & 20 & 6 & 32 & 6 \\
\hline $\begin{array}{l}\text { Dose of SCIG } \\
\text { (g/week) }\end{array}$ & 30 & 18 & 28 & 26.4 \\
\hline \multicolumn{5}{|c|}{$\begin{array}{l}\text { Isokinetic muscle strength at enrollment } \\
\text { (pet of norm) }\end{array}$} \\
\hline Wrist flexion trained/untrained & $29 / 31$ & $68 / 85$ & $78 / 71$ & $58 / 77$ \\
\hline Wrist extension trained/untrained & $38 / 48$ & $88 / 76$ & $69 / 120$ & $36 / 112$ \\
\hline Elbow flexion trained/untrained & $48 / 57$ & $111 / 95$ & $40 / 26$ & $63 / 94$ \\
\hline Elbow extension trained/untrained & $32 / 47$ & $57 / 100$ & $56 / 58$ & $13 / 79$ \\
\hline \multicolumn{5}{|l|}{$\begin{array}{l}\text { Aerobic capacity at enrollment } \\
\text { (pct of norm) }\end{array}$} \\
\hline $\mathrm{VO}_{2}-\mathrm{max}$ & 58 & 82 & 44 & 69 \\
\hline
\end{tabular}


A

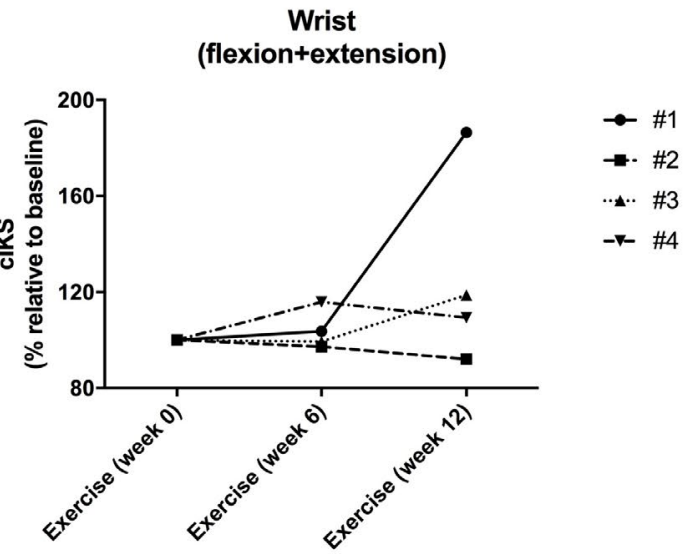

B

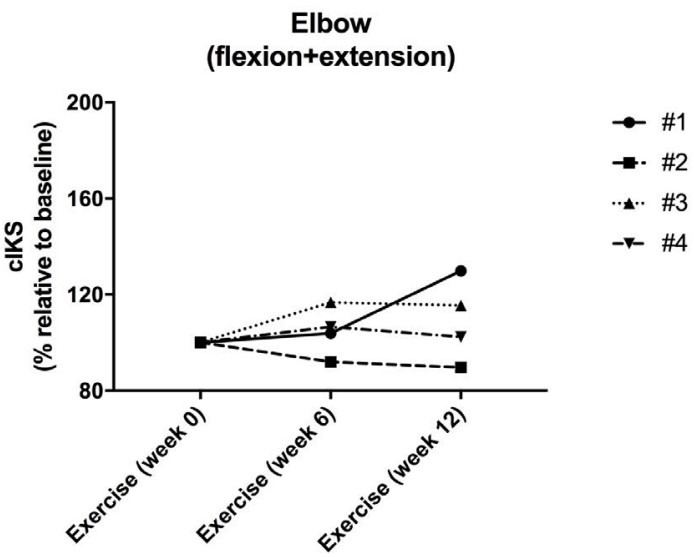

C

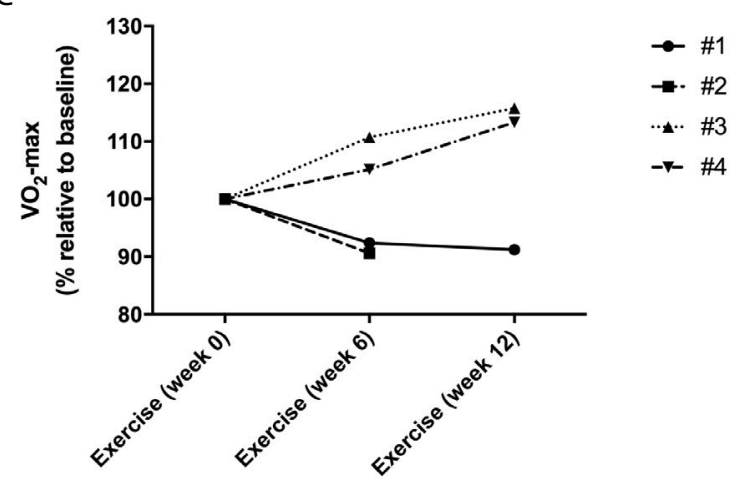

Figure 1. Individual changes in isokinetic muscle strength (cIKS) at wrist (A) and elbow (B) and the maximal oxygen consumption velocity $\left(\mathrm{VO}_{2}-\mathrm{max}\right)(\mathrm{C})$ relative to baseline level during the training period (from week 0 to 12 )

The low number of participants was due to only few patients accepting participation and the low prevalence of MMN, 0.6 to 2.0 per 100,000 (1) giving only up to 100 patients in Denmark. Some patients remain undiagnosed and others are treated with IVIG instead of SCIG. As IVIG is related to fluctuations in muscle strength between the infusions, only SCIG treated patients were eligible for inclusion in this study. The fraction of patients with MMN accepting and qualifying to participate (4 out of $15(28 \%)$ ) was similar to our study of exercise in CIDP [7].

Overall, the changes in muscle strength and aerobic capacity were inconsistent, but it is interesting that three of the four patients had an increase in muscle strength and only one had no effect. An increase of more than $10 \%$ on cIKS has previously been considered being clinical significant [11]. For the aerobic capacity two patients had increases of 13 and $15 \%$, whereas the other two had small decreases. As the weekly exercise sessions were unsupervised, we cannot be sure that the patients actually performed the exercise according to protocol. The use of diaries and regular contact by telephone or e-mail was the best way for us to encourage the participants to train sufficiently, and to increase load for resistance exercise. The self reported adherence to training was comparable to what we have previously shown for CIDP patients [7]. The MMN patients even increased their 1-RM more than CIDP patients, but at least one patient still did not obtain an increase in isokinetic muscle strength. Similarly, variable effects were shown for aerobic capacity after aerobic training. One explanation for the inconsistent findings is the low number of participants, but other possible explanations could be the long duration of disease in the MMN patients (13 years, range: 6 to 32 ), and the fact that $75 \%$ of the participants were older than 60 years. On the other hand, patient \#1 and \#3 have had MMN for 20 and 32 years and had both remarkable increase in muscle strength at both wrist ( $86 \%$ vs $18 \%$ ) and elbow ( $29 \%$ vs $17 \%$ ). Patient \#4 only had remarkable increase at wrist, and patient \#2 did not have increase at any time during the exercise period. He was also the only patient with a general higher muscle strength compared to the normal material. These findings indicate that it is possible to gain muscle strength at wrist and elbow despite most MMN patients are severely affected in these muscle groups.

Patient \#2 had an unexplained decrease in aerobic capacity during the run-in period of nearly $30 \%$ but did regain this prior to the start of the aerobic exercise period. Moreover, there was a decrease of muscle strength during the resistance exercise period. When evaluating this patient's training diary, it is clear that training was not performed three times weekly and that the increase in loads during the resistance period was insufficient. Finally, the patient dropped out of the study after eight weeks of aerobic exercise due to lower back pain.

Based on the reports of side effects from the patients, we find that resistance exercise in MMN is safe and feasible. As one patient developed back pain due to the aerobic training, and that the average aerobic capacity was unchanged it is unclear whether this is beneficial for MMN patients.

In conclusion, our findings show that resistance training is safe and may improve muscle strength at severely affected muscle groups. Aerobic capacity in patients with MMN is increased in some patients, but not in all and one patient developed lower back pain during aerobic exercise. These findings suggest that further and larger studies of exercise in MMN are needed.

\section{Ethical publication statement}

"We confirm that we have read the Journal's position on issues involved in ethical publication and affirm that this report is consistent with those guidelines."

\section{Disclosure of Conflicts of Interests}

Lars K Markvardsen received speaker honoraria from Octapharma

\section{Kristian Overgaard has no conflicts of interest}

John Vissing received research and travel support and/or speaker honoraria from Genzyme/Sanofi, Santhera Pharmaceuticals, aTyr 
Pharma and Ultragenyx Pharmaceuticals and served as consultant on advisory boards of Genzyme/Sanofi, aTyr Pharma, Ultragenyx Pharmaceuticals, Santhera Pharmaceuticals, Sarepta Therapeutics, Audentes Therapeutics, Stealth Biotherapeutics, NOVO Nordisk and Alexion Pharmaceuticals.

Henning Andersen received research and travel support from Octapharma, CSL Behring and

Genzyme/Sanofi, speaker honoraria from Genzyme/Sanofi and served as consultant on advisory board of UCB Pharma and NMD Pharma.

\section{References}

1. Cats EA, Jacobs BC, Yuki N, Tio-Gillen AP, Piepers S, et al. (2010) Multifocal motor neuropathy: association of anti-GM1 IgM antibodies with clinical features. Neurology 75: 1961-1967. [Crossref]

2. Elovaara I, Apostolski S, van Doorn P, Gilhus NE, Hietaharju A, et al. (2008) EFNS guidelines for the use of intravenous immunoglobulin in treatment of neurological diseases: EFNS task force on the use of intravenous immunoglobulin in treatment of neurological diseases. Eur J Neurol 15: 893-908. [Crossref]

3. Harbo T, Andersen H, Hess A, Hansen K, Sindrup SH, et al. (2009) Subcutaneous versus intravenous immunoglobulin in multifocal motor neuropathy: a randomized, single-blinded cross-over trial. Eur J Neurol 16: 631-638. [Crossref]
4. Garssen MP, Bussmann JB, Schmitz PI, Zandbergen A, Welter TG, et al. (2004) Physical training and fatigue, fitness, and quality of life in Guillain-Barre syndrome and CIDP. Neurology 63: 2393-2395. [Crossref]

5. Graham RC, Hughes RA, White CM (2007) A prospective study of physiotherapist prescribed community based exercise in inflammatory peripheral neuropathy. $J$ Neurol 254: 228-235. [Crossref]

6. Ruhland JL, Shields RK (1997) The effects of a home exercise program on impairment and health-related quality of life in persons with chronic peripheral neuropathies. Phys Ther 77: 1026-1039. [Crossref]

7. Markvardsen LH, Overgaard K, Heje K, Sindrup SH, Christiansen I, et al. (2018) Resistance training and aerobic training improve muscle strength and aerobic capacity in chronic inflammatory demyelinating polyneuropathy. Muscle Nerve 57: 70-76. [Crossref]

8. Harbo T, Andersen H, Overgaard K, Jakobsen J (2008) Muscle performance relates to physical function and quality of life in long-term chronic inflammatory demyelinating polyradiculoneuropathy. J Peripher Nerv Syst 13: 208-217. [Crossref]

9. Eriksen L, Gronbaek M, Helge JW, Tolstrup JS (2015) Cardiorespiratory fitness in 16 025 adults aged 18-91 years and associations with physical activity and sitting time. Scand J Med Sci Sports 26: 1435-1443. [Crossref]

10. Harbo T, Brincks J, Andersen H (2012) Maximal isokinetic and isometric muscle strength of major muscle groups related to age, body mass, height, and sex in 178 healthy subjects. Eur J Appl Physiol 112: 267-275. [Crossref]

11. Markvardsen LH, Sindrup SH, Christiansen I, Olsen NK, Jakobsen J, et al. (2017) Subcutaneous immunoglobulin as first-line therapy in treatment-naive patients with chronic inflammatory demyelinating polyneuropathy: randomized controlled trial study. Eur J Neurol 24: 412-418. [Crossref]

Copyright: (C2019 Markvardsen LK. This is an open-access article distributed under the terms of the Creative Commons Attribution License, which permits unrestricted use, distribution, and reproduction in any medium, provided the original author and source are credited. 